\title{
Minimierung des Sinterzerfalls mittels DEM
}

\author{
Michael Denzel und Michael Prenner
}

Lehrstuhl für Bergbaukunde, Bergtechnik und Bergwirtschaft, Montanuniversität Leoben, Leoben, Österreich

Eingegangen 26. November 2020; angenommen 21. Dezember 2020; online publiziert 27. Januar 2021

\begin{abstract}
Zusammenfassung: Um eine ausreichende Durchgasung im Hochofen zu gewährleisten, müssen feinkörnige Einsatzstoffe mithilfe des Sinterprozesses agglomeriert werden. Mechanische Belastungen an Förderanlagen, Kühlern, Sieben und Bunkern zwischen Sinteranlage und Hochofen führen zum teilweisen Zerfall des Sinters. Der dabei entstandene Feinkornanteil $(<5 \mathrm{~mm})$ muss vor der Beschickung des Hochofens ausgesiebt und als Rückgut unter hohem Energieaufwand, Kosten und entsprechenden CO2-Emissionen erneut der Sinteranlage zugeführt werden. Ziel der aktuellen Forschungsarbeit ist die Bereitstellung eines geeigneten Materialmodells, das es ermöglicht, den Zerfall von Hochofensinter während Transport und Lagerung mittels Diskrete Elemente Methode (DEM) vorherzusagen. Dies ermöglicht kritische Bereiche zu optimieren, um den Zerfall zu reduzieren. Dabei wird zuerst die Bruchcharakteristik des Sinters mittels eines eigens entwickelten Prüfstands für Einzelpartikelversuche analysiert. Mit den Versuchsergebnissen wird anschließend ein geeignetes Materialmodell in die DEM implementiert, welches auch in der institutseigenen Versuchsanlage verifiziert werden soll.
\end{abstract}

Schlüsselwörter: Hochofensinter, Bruchverhalten, Zerfall, DEM, Bruchmodell

Minimization of Sinter Degradation with DEM

Abstract: To ensure sufficient gas flow in the blast furnace, fine-grained input materials have to be agglomerated in the sinter process. Mechanical stress on several conveying systems, coolers, screens, and bunkers between sinter plant and blast furnace lead to degradation. Before the sinter is charged into the blast furnace, fines have to be screened out and sintered again with a high energy input, which causes high costs and $\mathrm{CO} 2$ emissions. The aim of this work is to provide an appropriate material model, which allows to pre-

Dipl.-Ing. M. Denzel ( $\bowtie)$

Lehrstuhl für Bergbaukunde, Bergtechnik und Bergwirtschaft,

Montanuniversität Leoben,

Franz-Josef-Straße 18,

8700 Leoben, Österreich

michael.denzel@unileoben.ac.at dict sinter degradation during transport and storage with DEM. This will allow to optimize critical conveying plants in order to minimize degradation. Therefore, the breakage behavior will be analyzed with an especially developed test rig for single-particle tests. With the test results, a material model will be implemented into DEM and verified in a test conveying plant.

Keywords: Blast furnace sinter, Breakage, Degradation, DEM, Breakage model

\section{Einleitung}

In der Sinteranlage werden vorerst feinkörniges Erz, Koks und weitere Hilfsstoffe in einer Mischtrommel vermengt. Der Sinterprozess selbst läuft auf einem sich langsam bewegenden Band ab, wobei die Mischung an der Oberfläche durch Zündbrenner gezündet wird und die heiße Prozessluft nach unten durch die Mischung gesaugt wird. Durch die hohen Temperaturen kommt es zum Anschmelzen der Eisenpartikel, welche sich somit verbinden. Am Ende des Sinterbandes wird der sogenannte Sinterkuchen durch Walzenbrecher zerkleinert. Partikel mit einer Korngröße kleiner als $5 \mathrm{~mm}$ werden abgesiebt und erneut der Sinteranlage zugeführt, weshalb dies allgemein als Rückgut bezeichnet wird. Anschließend wird der Sinter gekühlt, wobei hauptsächlich durch thermische Belastungen weiteres Rückgut anfällt und anschließend bei der Kaltabsiebung abgesondert wird. Insgesamt 30-40\% des gesamten Massenstromes fallen bei Heiß- und Kaltabsiebung als Rückgut an. Nach der Kaltabsiebung wird der Sinter üblicherweise als Fertigsinter bezeichnet und muss möglichst schonend zur Lagerstätte oder direkt zum Hochofen transportiert werden. Vor Beschickung des Hochofens wird der Sinter nochmals gesiebt, um weiteres entstandenes Rückgut aufgrund von Lagerung und Transport abzusondern (Abb. 1). Laut [1] werden vor der Hochofenbeschickung im EU-Durchschnitt $60 \mathrm{~kg}$ Rückgut pro Tonne Fertigsinter ausgesiebt und den weiten Weg zurück gefördert, um erneut der Sinteranlage zugeführt zu werden. Demnach werden aufgrund des Zerfalls 


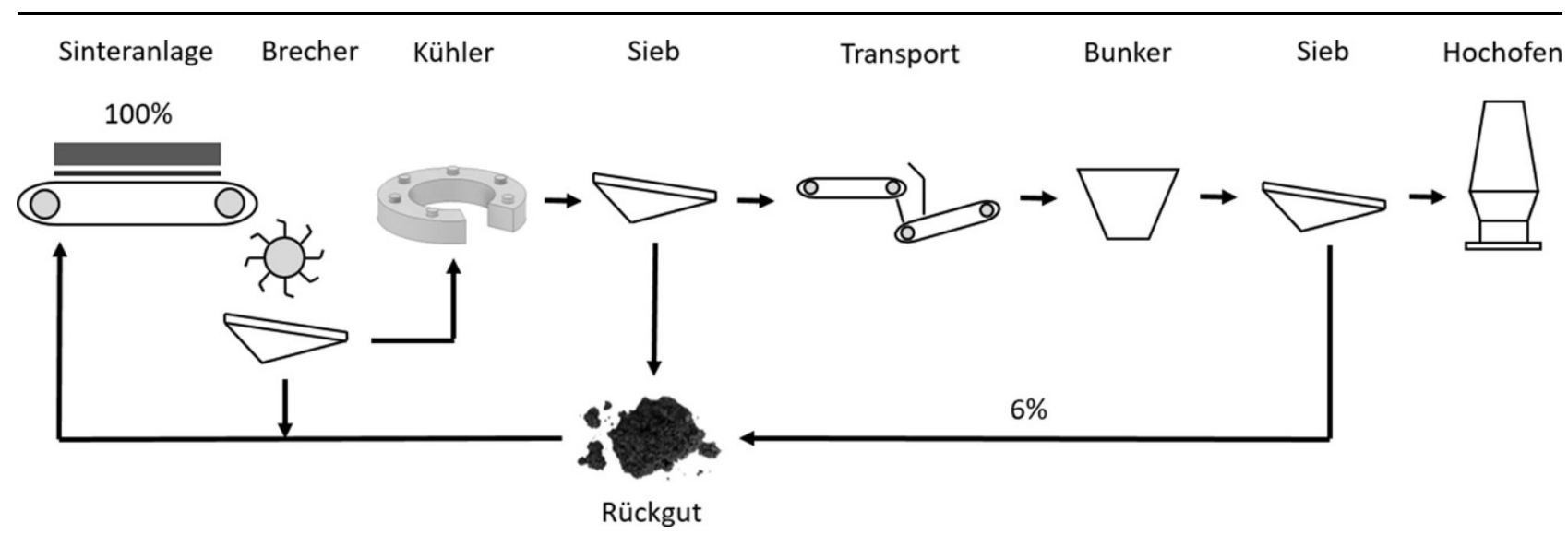

Abb. 1: Weg des Sinters

während Transport und Lagerung 6\% des Massenstroms im Kreis gefördert.

\section{Transport von Sinter}

Sinteranlage und Hochofen sind in der Regel einige hundert Meter voneinander entfernt. Zur Förderung des Sinters zwischen Sinteranlage und Hochofen werden hauptsächlich Gurtbandförderer verwendet. Aufgrund unterschiedlicher Lagerstätten, mehrerer Hochöfen und Zwischenspeicher sind einige Verzweigungen notwendig. Der Sinter durchläuft somit mehrere Schüttgutweichen und Schurren. Sehr häufig werden bei Übergabeschurren sogenannte "rock boxes" verwendet. Dabei werden bewusst Materialanhäufungen herbeigeführt, über die das folgende Material geleitet wird. Dies ist zwar günstig in Bezug auf Anlagenverschleiß, kann aber zu erhöhter Schüttgutdegradation führen (Abb. 2).

In den meisten Anlagen hat sich der Materialfluss gegenüber dem ursprünglich geplanten Massenstrom erhöht. Dies wurde hauptsächlich durch Erhöhung der Bandgeschwindigkeit erreicht. Zusätzlich wurden über die Jahre weitere Installationen vorgenommen, um z. B. Anlagenverschleiß zu minimieren, Proben zu entnehmen oder diverse Messungen durchzuführen. All dies führt häufig zu ungewolltem Materialflussverhalten, wie Materialanhäufungen

Abb. 2: Materialanhäufung an Übergabeschurre

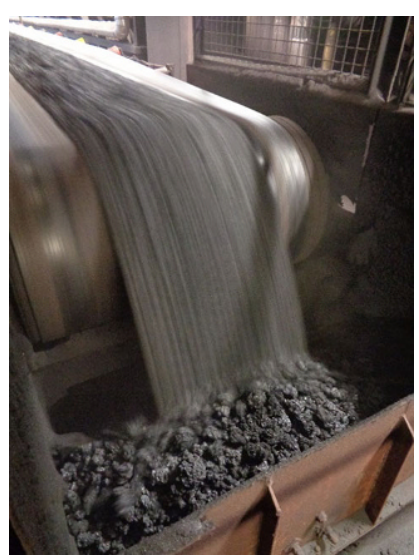

oder unnötigen Stößen gegen Schurrenwände, und fördert somit den Zerfall des Sinters.

\section{Lagerung von Sinter}

Hochofensinter wird entweder im Freien auf großen Schüttguthalden oder in Bunkern innerhalb der Anlage gelagert. Dies sind häufig quadratische Bunker, die mittels drehbaren Förderbands befüllt werden. Vor allem bei geringen Füllständen werden dabei große Fallhöhen überwunden, wobei es zu signifikantem Zerfall des Sinters kommt.

Des Weiteren kommt es zur Korngrößenentmischung. Beobachtungen und entsprechende DEM-Simulationen zeigen, dass sich größere Partikel in Förderrichtung des Beschickungsgurtbandförderers anhäufen (Abb. 3). Dies kann zu Schwankungen im Hochofenbetrieb führen.

\section{Charakterisierung des Bruchverhaltens}

Um ein geeignetes Materialmodell für die DEM bereitstellen zu können, muss vorerst die Bruchcharakteristik von Hochofensinter analysiert werden.

Standard zur Beurteilung der Festigkeit von Sinter und Koks ist heutzutage der nach ISO 556 genormte MICUMTest. Dabei wird eine definierte Schüttgutprobe in eine Stahltrommel gefüllt und die Partikelgrößenverteilung nach 100 Trommelumdrehungen mittels Siebanalyse festgestellt [2].

Eine weitere Methode zur Analyse der Festigkeit von Sinter und Koks ist der Shattertest. Dabei wird eine definierte Schüttgutprobe von einer bestimmten Höhe auf eine Stahlplatte fallen gelassen und ebenfalls die Partikelgrößenverteilung nach dem Fall bestimmt.

Laut Nistala [3] kann mit standardisierten Versuchsmethoden für die Sinterfestigkeit, wie Trommel- und Shattertest, der Einfluss der Transportparameter wie Fallhöhe und Förderlänge auf die Feinguterzeugung kaum ermittelt werden.

Standardisierte Prüfmethoden auf dem Gebiet der Sinterfestigkeit verwenden Schüttgutproben mit großer Partikelanzahl. Um das Bruchverhalten genauer zu untersu- 


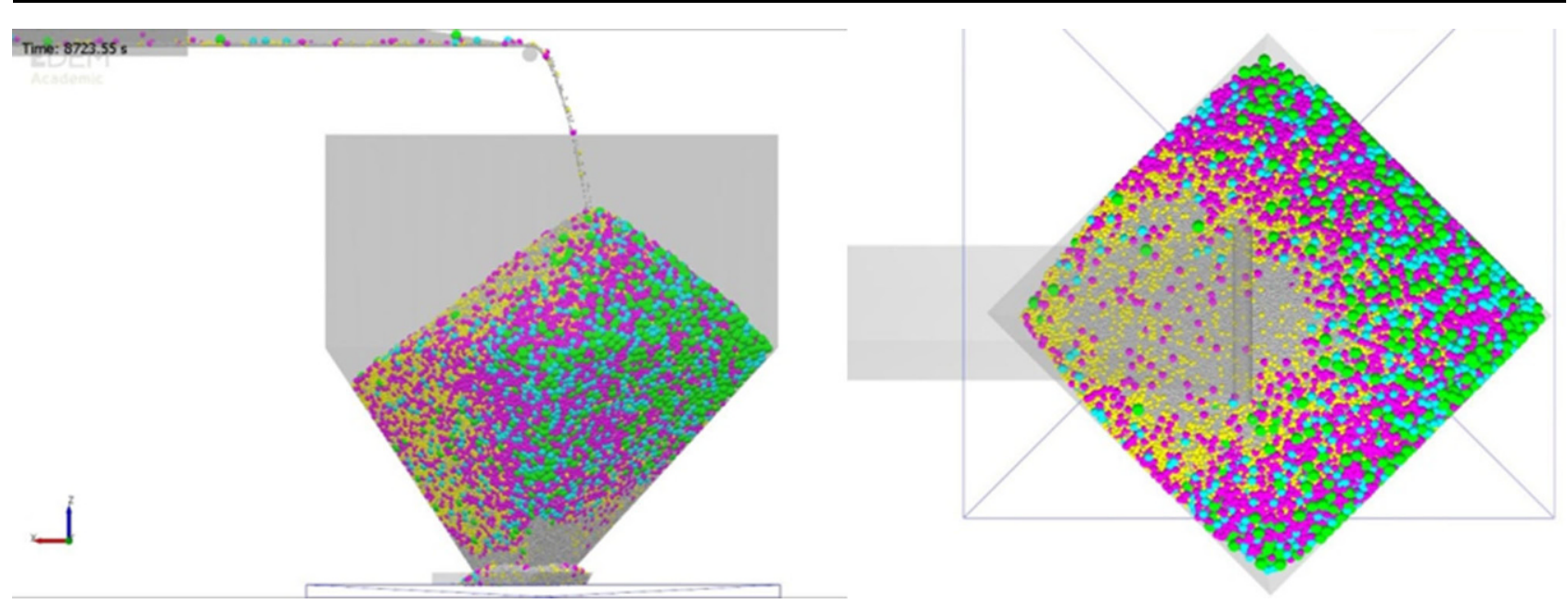

Abb. 3: Schüttgutentmischung in Bunker(Seitenansichtund Draufsicht). Partikelfraktionen-grau: bis $16 \mathrm{~mm}, g e l b:$ bis $25 \mathrm{~mm}, \mathrm{magenta:}$ bis $40 \mathrm{~mm}$, cyan: bis $50 \mathrm{~mm}$, grün: bis $100 \mathrm{~mm}$

chen, sind Einzelpartikeltests gefordert. In [4] wurde versucht, die Bruchcharakteristik von heißem Hochofensinter mittels Fallhammer zu charakterisieren. Zum Vergleich wurden ebenfalls Versuche in einem Backenbrecher durchgeführt. Aufgrund der nicht konsistenten Ergebnisse bezüglich Energieeintrag wurde dies mit zwei üblichen Gesteinsproben wiederholt, welche konsistente Ergebnisse lieferten. Die Untersuchungen zeigen einige Probleme bzw. Herausforderungen auf, wenn Prüfmethoden auf Materialien wie Hochofensinter mit stark variierenden Eigenschaften angewandt werden. Vor allem die große Heterogenität des Sinters macht eine Charakterisierung bei geringer Probenzahl unmöglich.

Traditionell wird die Größenreduktion beim Bruch in Abhängigkeit der Energie des Fallhammers nach Napier [5] angegeben. In [6] wurde eine Prüfmethodik zur Charakterisierung des Bruchverhaltens von Erzen in einem Partikelgrößenbereich von 13-45 mm entwickelt. Das Bruchmodell basiert dabei auf dem Modell von Vogel und Peukert [7], welches die mathematische Beschreibung der Bruchwahrscheinlichkeit als Weibull-Verteilung ermöglicht.

Zusammenfassend lässt sich festhalten, dass zwar viele Versuche mit einer Schüttgutprobe bzw. einer Vielzahl von Partikeln an Hochofensinter durchgeführt worden sind, bis dato aber keine Einzelpartikelbruchtests mit Fertigsin-

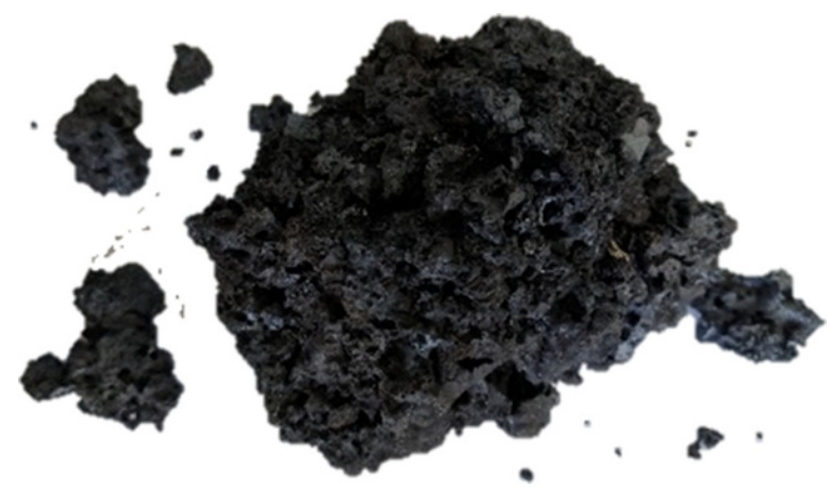

Abb. 4: Sinterpartikel mit Bruchfragmenten ter. Verschiedene Einzelpartikelbruchtests zur Analyse der Bruchcharakteristik für Erze und andere Mineralien sind bekannt. Ebenso ist die mathematische Beschreibung dieser möglich. Bis dato wurde dies allerdings nicht mit Hochofensinter durchgeführt, welcher sehr heterogene Eigenschaften aufweist (Abb. 4).

\section{Prüfstand für Hochofensinter}

Am Lehrstuhl für Bergbaukunde, Bergtechnik und Bergwirtschaft - Arbeitsgruppe Fördertechnik und Konstruktionslehre - wurde ein eigener Prüfstand speziell für Einzelpartikelversuche entwickelt. Dieser verfügt über zwei verschiedene Beschleunigungskonzepte, Verwiegung und Fragmentanalyse. Zudem ist dieser hochautomatisiert und ermöglicht, pro Minute einen Versuch durchzuführen. Ziel der Versuche ist es, sowohl die Bruchwahrscheinlichkeit als auch die Partikelgrößenverteilung nach dem Bruch in Abhängigkeit der eingebrachten Prüfenergie zu analysieren (Abb. 5).

Bei der Prüfmethodik zur Charakterisierung des Bruchverhaltens von Hochofensinter ergeben sich mehrere Herausforderungen. Der häufigste Belastungsfall während des Transports von Sinter ist der Stoß gegen eine Schurrenwand oder einen anderen Partikel. Um dies möglichst realitätsgetreu nachbilden zu können, müssen Prallversuche durchgeführt werden. Ein Hammerschlag wäre aufgrund der zwei Kontaktflächen und der unterschiedlichen Spannungsverteilung im Partikel nur schwierig zu vergleichen. Beim Prallversuch muss der Sinter möglichst schonend beschleunigt werden, um Partikelbruch vor dem eigentlichen Versuch zu vermeiden. Üblicherweise werden Sinterpartikel in Größen von 5-150 mm zum Hochofen transportiert. Die Festigkeit des Sinters ist größenabhängig, d.h. große Partikel neigen allgemein eher zum Bruch als kleinere. Um die Energiebereiche für einen sicheren Bruch bzw. keinen Bruch bei verschiedenen Partikelgrößen festzustellen, wurde eine Voruntersuchung mit einfachen Fallversuchen durchgeführt. 
Abb. 5: Speziell entwickelter Prüfstand für automatisierte Einzelpartikelbruchversuche
Abb. 6: Schüttgutsimulation mittels DEM an einer Schurre für Hochofensinter
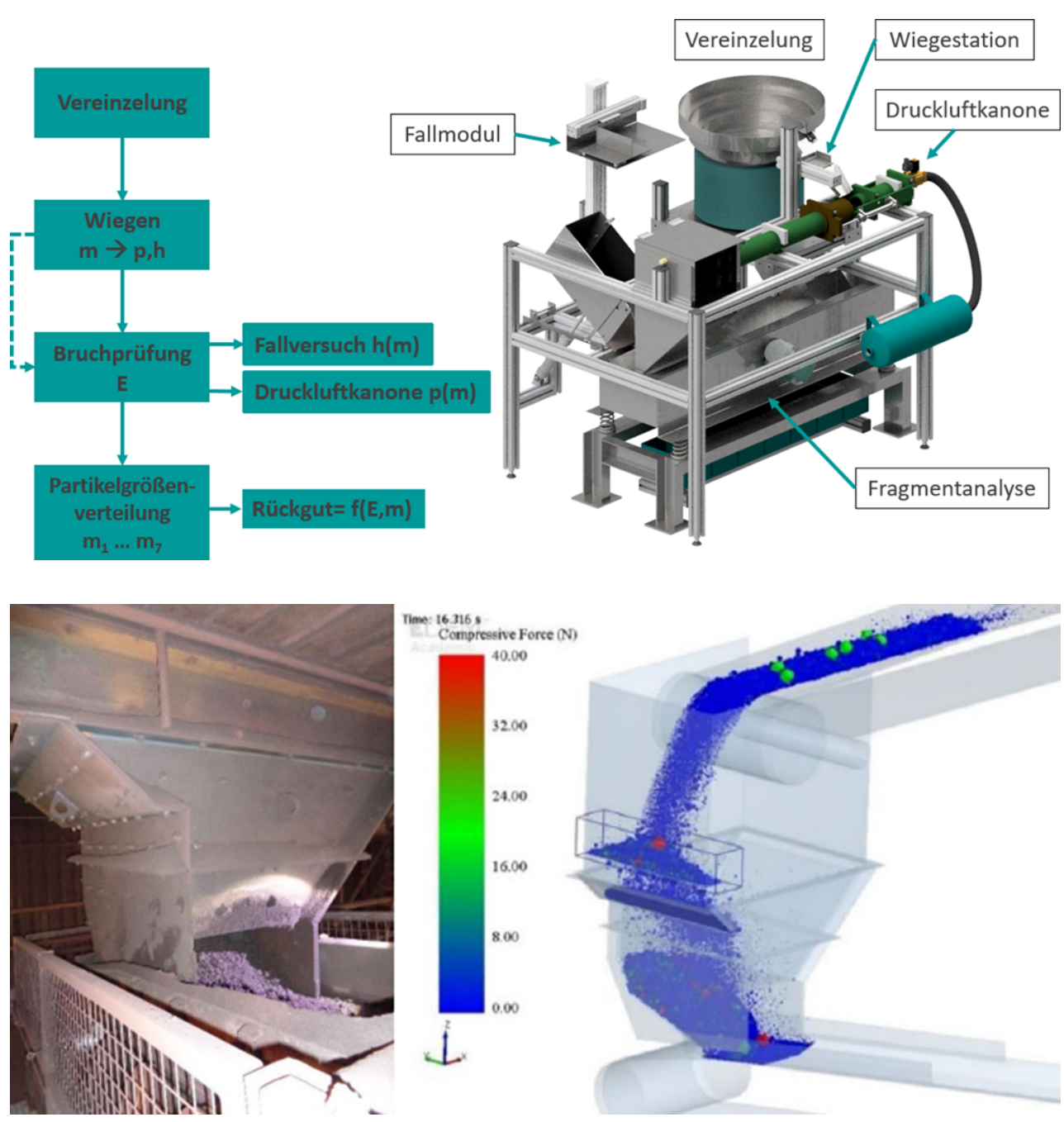

Die Versuche ergaben, dass abhängig von der Partikelgröße sehr unterschiedliche Prüfenergien nötig sind. Solch große Energiebereiche wären mit konventionellen Prüfmethoden, wie Fallversuch oder Fallhammer, ohnehin nur schwierig umsetzbar. Im eigens entwickelten Prüfstand für Hochofensinter wird für kleine Energien ein Fallversuch durchgeführt und für größere Energien werden die Sinterpartikel mittels Druckluftkanone beschleunigt. Des Weiteren verlangt die Heterogenität und die geometrisch undefinierte Form des Sinters nach einer großen Probenzahl, um anhand gemittelter Werte eine Aussage über die Bruchcharakteristik tätigen zu können. Bei konventionellen Prüfmethoden wird nach dem Bruchversuch eine Siebanalyse mittels Siebturm durchgeführt. Dies könnte zu weiterem Partikelbruch führen und wäre zu aufwendig für größere Probenzahlen, weshalb eigens eine automatische Fragmentanalyse entwickelt wurde. Dabei kann die Partikelgrößenverteilung sowohl schneller als auch schonender als mittels Sieben festgestellt werden. Dies ermöglicht es, jede Minute einen Versuch durchführen zu können. Zur noch effizienteren Versuchsdurchführung wird derzeit eine automatische Beladung der Druckluftkanone implementiert. Diese wird zusätzlich über eine automatische
Verwiegung und Vereinzelung verfügen. Ziel ist es, den Prüfstand nur noch mit einer Schüttgutprobe anstatt mit jeweils einzelnen Partikeln beschicken zu müssen.

\section{DEM-Modell}

Zur Anlagenoptimierung wurden einige Schüttgutsimulationen mit Hochofensinter durchgeführt (Abb. 6). Diese wurden bisher mit kugelförmigen Standard-Partikeln durchgeführt und waren auf übliche Schüttgutparameter, wie Schüttwinkel und Wandreibung, kalibriert. Dabei konnte das Materialflussverhalten gut abgebildet werden, es können aber keine Aussagen über die Feinguterzeugung getroffen werden. Lokale Belastungen können zwar über Ausgabe der Druckkraft auf die Partikel visualisiert werden, zur Abschätzung der Feinguterzeugung ist dies allerdings nicht ausreichend.

Mit den Ergebnissen der Einzelpartikelbruchversuche soll demnach ein Materialmodell der DEM modifiziert und, wenn nötig, erweitert werden, um den Sinterbruch und somit die Feinguterzeugung simulieren zu können. Aktuell gibt es drei verschiedene Partikelbruchmodelle in der DEM, 
welche in [8] miteinander verglichen wurden: bonded-particle-model (BPM), particle-replacement-model (PRM) und fast-breakage-model (FBM). Beim BPM von Potyondy und Cundall [9] wird ein Partikel aus mehreren kleinen Partikeln aufgebaut, die mit einem virtuellen Zylinder verbunden sind. Sobald dieser masselose Zylinder eine definierte kritische Spannung erfährt, wird die Verbindung aufgebrochen. Das BPM eignet sich besonders für brüchiges Material und wurde in der Arbeit von Khanal und Schubert [10] für hochfeste Agglomerate verwendet. Nachteilig beim BPM ist jedoch der relativ hohe Rechenaufwand im Vergleich zum PRM. Auch in [11] wurde das BPM erfolgreich eingesetzt, um den Partikelbruch von Granit in einem Kegelbrecher zu simulieren. Aufgrund des hohen Rechenaufwands wurde allerdings nur ein Viertel davon simuliert.

Das fast-breakage-model (FBM) beschreibt laut Jimenez [8] die Partikelgrößenverteilung nach dem Bruch nicht ausreichend und ist daher für die Vorhersage der Feinguterzeugung bei Hochofensinter weniger geeignet.

Beim particle-replacement-model (PRM) wird ein Partikel bei Erfüllung des Versagenskriteriums durch viele kleinere ersetzt. Es liefert eine realitätsnahe Partikelgrößenverteilung nach dem Bruch und erfordert vor allem weniger Rechenleistung als das BPM. Nachteilig beim PRM ist allerdings der Massen- bzw. Volumenverlust, der entsteht, wenn eine große Kugel durch viele kleinere ersetzt wird. In [12] wurde das PRM eingesetzt, um die Degradation von Kupfererz in einer Trommelmühle zu simulieren. Der Massenverlust wurde dabei mittels Erhöhung der Dichte ausgeglichen, wobei man ein immer schwerer und feiner werdendes Pulver erhält. Die Änderung der Schüttdichte wäre für Schüttgutsimulationen in Anlagen sehr problematisch, da somit das Belastungsverhalten nicht mehr realitätsgetreu simuliert werden könnte.

Aufgrund des Massenerhalts wird vorerst der Ansatz des bonded-particle-model (BPM) für die Schüttgutsimulation von Hochofensinter priorisiert. Entscheidender Punkt bei der Implementierung dieses Materialmodells ist das Versagenskriterium des verbindenden virtuellen Zylinders. Ein erster Ansatz ist die Variation der kritischen Spannung der virtuellen Zylinder, bis die Bruchwahrscheinlichkeit und Partikelgrößenverteilung nach dem Bruch in der Simulation mit den Versuchsergebnissen übereinstimmen. Die Kalibration kann dabei durch Simulation des Prallversuchs durchgeführt werden (Abb. 7). Sollte dies nicht ausreichen, um das komplexe Bruchverhalten von Hochofensinter ausreichend nachbilden zu können, muss das Materialmodell entsprechend modifiziert oder erweitert werden.

Abb. 7: Simulation des Prallversuchs zur Kalibration des bonded-particle-models

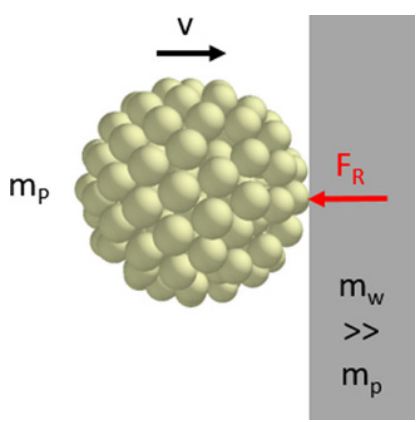

Zur Verifizierung des Materialmodells sind Versuche im eigenen Förderkreislauf der Arbeitsgruppe Fördertechnik geplant, bei denen das entstandene Feingut mit den Simulationsergebnissen verglichen wird.

\section{Ausblick}

Um Schüttgutsimulationen mit dem in dieser Forschungsarbeit angestrebten Materialmodell durchführen zu können, erfordert es viel Rechenleistung. Mit zunehmender Rechenleistung wird es möglich sein, noch genauere Partikelbruchsimulationen effizient durchzuführen und ganze Anlagen bezüglich Sinterzerfall zu optimieren. Bei einer durchschnittlichen Jahresproduktion von 93 Mio. t Sinter in der EU und durchschnittlichen Prozesskosten von $20 € /$ t Rückgut würde es neben massiven Energie- und CO2-Einsparungen ein EU-weites Einsparungspotential von 18,6 Mio. € jährlich bedeuten, falls man den Sinterzerfall nur um $1 \%$ reduzieren könnte [1].

Funding. Open access funding provided by Montanuniversität Leoben.

Open Access Dieser Artikel wird unter der Creative Commons Namensnennung 4.0 International Lizenz veröffentlicht, welche die Nutzung, Vervielfältigung, Bearbeitung, Verbreitung und Wiedergabe in jeglichem Medium und Format erlaubt, sofern Sie den/die ursprünglichen Autor(en) und die Quelle ordnungsgemäß nennen, einen Link zur Creative Commons Lizenz beifügen und angeben, ob Änderungen vorgenommen wurden.

Die in diesem Artikel enthaltenen Bilder und sonstiges Drittmaterial unterliegen ebenfalls der genannten Creative Commons Lizenz, sofern sich aus der Abbildungslegende nichts anderes ergibt. Sofern das betreffende Material nicht unter der genannten Creative Commons Lizenz steht und die betreffende Handlung nicht nach gesetzlichen Vorschriften erlaubt ist, ist für die oben aufgeführten Weiterverwendungen des Materials die Einwilligung des jeweiligen Rechteinhabers einzuholen.

Weitere Details zur Lizenz entnehmen Sie bitte der Lizenzinformation auf http://creativecommons.org/licenses/by/4.0/deed.de.

\section{Literatur}

1. BAT - reference document IED 2010/75/EU, 2012

2. ISO 556 - Coke (greater than $20 \mathrm{~mm}$ in size) - Determination of mechanical strength, 2020

3. Nistala, S. H.; Sinha, M.; Choudhary, M. K.; Bose, G.; Sinha, S. Study of generation of sinter return fines during transportation, Ironmaking \& Steelmaking, 42 (2015), Nr. 3, S. 226-232, https://doi. org/10.1179/1743281214Y.0000000224

4. Öfner, W.; Zaunrith, G.: The Drop Weight Test Revisited: Characterization of the Crushability of Hot Sinter and Validation of the Approach with Natural Rocks, BHM, 161 (2016), Nr. 6, S. 277-282, DOI https://doi.org/10.1007/s00501-016-0501-7

5. Napier-Munn, T. J.; Morrell, S.; Morrison, R. D.; Kojovic, T.: Mineral comminution circuits: Their operation and optimization, Indooroopilly, Qld Australia: Julius Kruttschnitt Mineral Research Centre, University of Queensland, 1996

6. Zuo, W.; Shi, F.: Ore impact breakage characterisation using mixed particles in wide size range. In: Minerals Engineering, 86 (2016), S. 96-103, https://doi.org/10.1016/j.mineng.2015.12.007

7. Vogel, L.; Peukert, W.: Breakage behaviour of different materials-Construction of a master curve for the breakage pro bability. Powder Technol., 129 (2003), S. 101-110, https://doi.org/10.1016/ S0032-5910(02)00217-6 
8. Jiménez-Herrera, N.; Barrios, G. K. P.; Tavares, L. M.: Comparison of breakage models in DEM in simulating impact on particle beds, Advanced Powder Technology, 29 (2018), Nr. 3, S. 692-706, https:// doi.org/10.1016/j.apt.2017.12.006

9. Potyondy, D. O.; Cundall, P. A.: A bonded-particle model for rock, Int. J. Rock Mech. \& Min. Sci., 41 (2004), Nr. 8, S. 1329-1364, https://doi. org/10.1016/j.ijrmms.2004.09.011

10. Khanal, M.; Schubert, W.; Tomas, J.: Oblique impact simulations of high strength agglomerates, Advanced Powder Technology, 20 (2009), S. 150-157, https://doi.org/10.1016/j.apt.2008.06.00.
11. Quist, J.; Evertsson, C. M.: Cone crusher modelling and simulation using DEM, Minerals Engineering, 85 (2016), S. 92-105, https://doi. org/10.1016/j.mineng.2015.11.004

12. Sousani, M.; Chagas, A.; Saxena, A.; Yang, Y.: Simulation of Surface Damage and Body Breakage by using DEM, NAFEMS, 2019

Hinweis des Verlags. Der Verlag bleibt in Hinblick auf geografische Zuordnungen und Gebietsbezeichnungen in veröffentlichten Karten und Institutsadressen neutral. 